\title{
Ellipsoid Fitting Calibration Method Based on Genetic Algorithm
}

\author{
CHEN Genpu ${ }^{1, a}$, LEI Xusheng ${ }^{1, b}$ and GENG Yue ${ }^{1, c}$ \\ ${ }^{1}$ School of Instrument Science and Opto-electronics Engineering, Beihang University, Beijing \\ 100191, China \\ achengenpu1992@163.com, yushangtianxia@163.com, coybuaa@163.com
}

\begin{abstract}
Keywords: magnetic compass calibration, ellipsoid fitting, ellipse constraint, genetic algorithm, eigenvector.

Abstract. To calibrate three-axis magnetic compass in complex conditions, an ellipsoid fitting algorithm is proposed in this paper, which is based on genetic algorithm searching for intersecting plane. First, by intersecting ellipsoid surface with a plane equation covering the whole space, the corresponding elliptic equation could be obtained. Then, using minimum sum of squares of the distance between data points and the fitting ellipsoid as the index, the three-dimensional magnetic compass data are fitted with the ellipse constraint. The adoption to the genetic algorithm is followed to search for the optimal intersecting plane equation, thus obtaining the optimal correction parameters of magnetic compass. Based on a series of tests, the static azimuth accuracy and dynamic azimuth accuracy can reach 0.46 degree and 0.68 degree respectively. This proposed method has high calibration accuracy and can meet the application requirements of magnetic compass.
\end{abstract}

\section{Introduction}

In recent years, the Unmanned Aerial Vehicle (UAV) has shown high potential in civil application, such as high voltage inspection, line inspection, etc. With the magnetic compass, the UAV can generate the corresponding azimuth information to finish task under GPS losing environment ${ }^{[1]}$. However, the magnetic compass has bias, scale factor error and non-orthogonal error ${ }^{[2]}$. In addition, magnetic compass is easily interfered by surrounding ferromagnetic materials, which is mainly divided into hard-iron interference and soft-iron interference ${ }^{[2]}$. Therefore, uncalibrated magnetic compass can not provide the corresponding azimuth information for the UAV.

Based on external azimuth reference, Browditch proposed the compass compensation algorithm approximate the deviation to the low-order Fourier series of azimuth, and then compensate the magnetic azimuth ${ }^{[3][4]}$. But it requires accurate azimuth information, which is not applicable in many situations $^{[5]}$. YUN JaeMu et al. adopted maximum-minimum method to calibrate the magnetic compass $^{[6]}$. By rotating the magnetic compass at any two planes of the body coordinate, the maximum value and minimum value of the magnetic field in each axis can be acquired to get calibration parameters. In spite of the advantages of simplicity and easy operation, the calibration precision is limited because this method just considers the bias and scale factor error. In addition, the calibration parameters which are obtained by the maximum value and minimum value, are sensitive to the measurement noise. Dorveaux Eric et al. proposed a linear iterative calibration method based on constraints $^{[7]}$. It assumes that the norm of three-axis magnetic field is 1 , which is used as the constraint. Then with the index that the minimum sum of square difference between the norm of magnetic field and 1 , the calibration parameter is solved by the least square method. However, the accuracy of linear iterative method depends on the initial value and the calculation is very complex.

Considering the influence of error and interference, the data of the triaxial magnetic compass are actually distributed on an ellipsoid. Therefore, the ellipsoid fitting is an effective magnetic compass calibration method for the magnetic compass measurement. In the literature [8], an ellipse fitting method with constraint is proposed. The minimum sum of squares of the distance between data points and the fitting elliptic curve is chosen as the index, and then the data of magnetic compass are fitted. But this ellipse fitting method is not available for the triaxial magnetic compass. In the literature [9], an ellipsoid fitting calibration method with a constraint is proposed. But the constraint is actually the 
necessary and sufficient condition of ellipse fitting ${ }^{[10]}$. By intercepting elliptic surface with a plane equation, an elliptic curve is got and the parameters of elliptic equation can be calculated with the ellipse constraint. However, the plane is easily leads to the solution that is non-optimal ${ }^{[11]}$.

To get optimal solution, a new magnetic compass calibration method which searches the optimal intersecting plane equation is proposed. Using the minimum sum of distance between the data and fitting ellipsoid as index, the optimal ellipsoid parameters can be calculated with the optimal intersecting plane equation searched by genetic algorithm. Finally the calibration parameter can be obtained from the ellipsoid parameters.

The overall layout of this paper is defined as follows: Section II gives the detailed descriptions of the error model and the ellipsoid equation of magnetic sensors. Section III proposes the calibration algorithm based on genetic algorithm searching for intersecting plane. Section IV verifies the effectiveness of the method by experiments, and the conclusion is given in Section V.

\section{Magnetic Compass Measurement Model}

The measurements of magnetic compass are the projections of magnetic field vector on the body axis and are denoted as a $\boldsymbol{m}_{b} \in R^{3 \times 1}$. Meanwhile, the measurements of the error-free magnetic compass under no interference are denoted as $\boldsymbol{m}_{n} \in R^{3 \times 1}$. To describe the relationship between $\boldsymbol{m}_{b}$ and $\boldsymbol{m}_{n}$ with measurement errors, the magnetic compass measurement model is shown as follows:

$$
\boldsymbol{m}_{b}=\boldsymbol{K}_{\mathrm{S}} \boldsymbol{K}_{\mathrm{N}} \boldsymbol{m}_{n}+\boldsymbol{b}_{1}+\boldsymbol{w}
$$

where $\boldsymbol{K}_{\mathrm{S}}$ is a 3-D diagonal matrix that represents the influence of scale factor error. $\boldsymbol{K}_{\mathrm{N}}$ is a 3-D matrix that indicates the non-orthogonal error result from the magnetic compass itself. $\boldsymbol{b}_{1}$ and $\boldsymbol{w}$ are both 3-D vectors, representing bias and white noise respectively.

In practical applications, surrounding ferromagnetic materials can interfere with magnetic compasses, which mainly include hard magnetic interference and soft magnetic interference. The influence of hard-iron interference can be equivalent to the bias of the magnetic compass, and the influence of soft-iron disturbance can be equivalent to the scale factor error. Taking into account the influence of external interference, the measurement model of magnetic compass can be expressed as follows:

$$
\boldsymbol{m}_{b}=\boldsymbol{K}_{\mathrm{S}} \boldsymbol{K}_{\mathrm{N}} \boldsymbol{K}_{\mathrm{sf}} \boldsymbol{m}_{n}+\boldsymbol{b}_{\mathrm{m}}+\boldsymbol{w}=\boldsymbol{K} \boldsymbol{m}_{n}+\boldsymbol{b}_{\mathrm{m}}+\boldsymbol{w}
$$

Where $\boldsymbol{K}_{\mathrm{sf}}$ is a 3-D diagonal matrix that represents the interference of soft-iron. $\boldsymbol{b}_{\mathrm{m}}$ is a vector of 3-D which represents the equivalent bias of the integration of magnetic compass's bias and hard-iron interference. The purpose of the calibration is to determine the matrix $\boldsymbol{K}$ and the vector $\boldsymbol{b}_{\mathrm{m}}$, and then to calculate the theoretical measurement value $\boldsymbol{m}_{n}$ by the measurement value $\boldsymbol{m}_{b}$.

The theoretical measurements of magnetic compass $\boldsymbol{m}_{n}$ remain constant when the magnetic compass is only rotating at fixed points. This face can be expressed as follows:

$$
\left\|\boldsymbol{m}_{n}\right\|=\sqrt{m_{n x}{ }^{2}+m_{n y}{ }^{2}+m_{n z}{ }^{2}}=\text { const }
$$

When rotating the magnetic compass in three dimensional space, the sampling points of the magnetic compass are distributed on the sphere of which the center is at the origin. Substituting Eq. (2) into to Eq. (3) can lead to 


$$
\left(\boldsymbol{m}_{b}\right)^{\mathrm{T}} \boldsymbol{H} \boldsymbol{m}_{b}-2 \boldsymbol{b}_{\mathrm{m}}^{\mathrm{T}} \boldsymbol{H} \boldsymbol{m}_{b}+\boldsymbol{b}_{\mathrm{m}}^{\mathrm{T}} \boldsymbol{H} \boldsymbol{b}_{\mathrm{m}}+W=\text { const }
$$

where $\boldsymbol{H}=\boldsymbol{G}^{\mathrm{T}} \boldsymbol{G}, \boldsymbol{G}=\boldsymbol{K}^{-1}=\operatorname{chol}(\boldsymbol{H}), W=\left[2\left(\boldsymbol{m}_{b}-\boldsymbol{b}_{\mathrm{m}}\right)+\boldsymbol{w}\right]^{\mathrm{T}} \boldsymbol{G}^{\mathrm{T}} \boldsymbol{G} \boldsymbol{w}$.

The ellipsoid fitting method can provide sufficient accurate parameters when $w$ tends to $0^{[12]}$. Eq. (4) is the quadratic form of the ellipsoid. It is obvious that the data points collected by the magnetic compass at the fixed point are distributed on the ellipsoid, whose center deviates from the origin due to its own error and the influence of external disturbances.

The parameters of quadratic expressions are assumed as follows:

$$
\begin{aligned}
& \boldsymbol{H}=\left[\begin{array}{ccc}
a & d / 2 & f / 2 \\
d / 2 & b & e / 2 \\
f / 2 & e / 2 & c
\end{array}\right] \\
& \boldsymbol{b}_{\mathrm{m}}=-\frac{1}{2} \boldsymbol{H}^{-1}\left(\begin{array}{l}
h \\
i \\
j
\end{array}\right)
\end{aligned}
$$

Substituting Eq. (5) and Eq. (6) into Eq. (4) can lead to

$$
a m_{b x}^{2}+b m_{b y}^{2}+c m_{b z}^{2}+d m_{b x} m_{b y}+e m_{b y} m_{b z}+f m_{b x} m_{b z}+h m_{b x}+i m_{b y}+j m_{b z}+l=0
$$

\section{Ellipsoid Fitting Calibration Method Based on Genetic Algorithm Searching for the Optimal Intersecting Plane Equation}

Ellipsoid Fitting Based on Plane Intersection. The ellipsoid fitting method of plane intersection is to intersect ellipsoid with a plane, and get the ellipse firstly. And then, the 3-D ellipsoid data are fitted by combining with the ellipse fitting constraint. Finally the parameters of ellipsoid can be obtained. Therefore, the constraint in the literature [11] can be applied to the ellipsoid fitting method by adopting the fitting method of plane intersection.

The plane equation can be expressed as follows:

$$
m_{b z}=\alpha m_{b x}+\beta m_{b y}+\gamma
$$

where $\alpha, \beta$ and $\gamma$ are all certain constant.

Substituting Eq. (8) into the ellipsoid Eq. (7), the elliptic equation can be described as

$$
A m_{b x}^{2}+B m_{b y}^{2}+C m_{b x} m_{b y}+D m_{b x}+E m_{b y}+F=0
$$

where

$$
\begin{aligned}
& A=a+\alpha^{2} c+\alpha f \\
& B=b+\beta^{2} c+\beta e \\
& C=2 \alpha \beta c+d+\alpha e+\beta f
\end{aligned}
$$

The ellipse fitting constraint $4 A B-C^{2}=1$ can be written in matrix form

$$
\boldsymbol{k}^{\mathrm{T}} \boldsymbol{M} \boldsymbol{k}=1
$$

where 


$$
\begin{aligned}
& \boldsymbol{k}=\left[\begin{array}{llllllllll}
a & b & c & d & e & f & h & i & j & l
\end{array}\right]^{\mathrm{T}} \\
& \boldsymbol{M}=\left[\begin{array}{ll}
\boldsymbol{M}_{11} & \boldsymbol{0}_{6 \times 4} \\
\boldsymbol{O}_{4 \times 6} & \boldsymbol{0}_{4 \times 4}
\end{array}\right] \\
& \boldsymbol{M}_{11}=\left[\begin{array}{cccccc}
0 & 2 & 2 \beta^{2} & 0 & 2 \beta & 0 \\
2 & 0 & 2 \alpha^{2} & 0 & 0 & 2 \alpha \\
2 \beta^{2} & 2 \alpha^{2} & 0 & -2 \alpha \beta & 0 & 0 \\
0 & 0 & -2 \alpha \beta & -1 & -\alpha & -\beta \\
2 \beta & 0 & 0 & -\alpha & -\alpha^{2} & \alpha \beta \\
0 & 2 \alpha & 0 & -\beta & \alpha \beta & -\beta^{2}
\end{array}\right]
\end{aligned}
$$

Combined objective function and constraint Eq. (11), the Lagrangian multiplier method is used to calculate the ellipsoid fitting parameters.

An Ellipsoid Fitting Equation Based on Constraint. It is necessary to determine the objective function before fitting the data of the triaxial magnetic compass. Due to the fact that the actual magnetic compass output data only approximately satisfy the Eq. (7), the function $g$ can be expressed as follows according to Eq. (7):

$$
g(\boldsymbol{m}, \boldsymbol{k})=a m_{b x}^{2}+b m_{b y}^{2}+c m_{b z}^{2}+d m_{b x} m_{b y}+e m_{b y} m_{b z}+f m_{b x} m_{b z}+h m_{b x}+i m_{b y}+j m_{b z}+l=\boldsymbol{m} \cdot \boldsymbol{k}
$$

where

$$
\boldsymbol{m}=\left[m_{b x}^{2}, m_{b y}^{2}, m_{b z}^{2}, m_{b x} m_{b y}, m_{b y} m_{b z}, m_{b x} m_{b z}, m_{b x}, m_{b y}, m_{b z}, 1\right]^{\mathrm{T}}
$$

Since $g\left(\boldsymbol{m}_{i}, \boldsymbol{k}\right)$ is the distance from the data point $\boldsymbol{m}_{i}$ to the surface of the fitting ellipsoid, the objective function is constructed as

$$
\min \sum_{i=1}^{N} g\left(\boldsymbol{m}_{i}, \boldsymbol{k}\right)=\|\boldsymbol{J k}\|^{2}
$$

where $\boldsymbol{J}=\left[\begin{array}{r}\boldsymbol{m}_{1} \\ \boldsymbol{m}_{2} \\ \mathrm{M} \\ \boldsymbol{m}_{N}\end{array}\right] . N$ represents the number of data points.

If the plane of the ellipsoid is arbitrarily selected, the corresponding parameters of the ellipsoid are not optimal. Considering that the coefficient of $m_{b z}$ is -1 in Eq. (8), if $\alpha$ and $\beta$ are set range region in genetic algorithm, the ratio of $\alpha, \beta$ and -1 is in a certain range. Therefore the plane range represented by the equation is limited. To solve this problem, a new plane equation which makes the representation of the plane equation cover the whole space is proposed as follows:

$$
\alpha m_{b x}+\beta m_{b y}+\gamma m_{b z}+z=1
$$

And then, the plane Eq. (18) can be transformed into

$$
m_{b z}=-\frac{\alpha}{\gamma} m_{b x}-\frac{\beta}{\gamma} m_{b y}-\frac{z}{\gamma}
$$


It is clear that the Eq. (19) do not include the plane $\gamma=0$. Thus, a way to process this problem is presented as follows:

$$
\begin{aligned}
& L_{\mathrm{m}}=\max \{|\alpha| \quad|\beta| \quad|\gamma|\} \\
& \begin{cases}m_{b x}=-\frac{\beta}{\alpha} m_{b y}-\frac{\gamma}{\alpha} m_{b z}-\frac{z}{\alpha} & L_{\mathrm{m}}=|\alpha| \\
m_{b y}=-\frac{\alpha}{\beta} m_{b x}-\frac{\gamma}{\beta} m_{b z}-\frac{z}{\beta} & L_{\mathrm{m}}=|\beta| \\
m_{b z}=-\frac{\alpha}{\gamma} m_{b x}-\frac{\beta}{\gamma} m_{b y}-\frac{z}{\gamma} & L_{\mathrm{m}}=|\gamma|\end{cases}
\end{aligned}
$$

According to Eq. (20), the Eq. (18) can be processed and taken into Eq. (7), and then the different forms of elliptic equations will be obtained. The matrix $\boldsymbol{M}$ of Eq. (11) is also different, and is directly related to the plane parameter $\alpha, \beta$ and $\gamma$. In this way, it can be guaranteed that the plane Eq. (20) that genetic algorithm searches for region covering all the planes of the 3-D space.

Thus, combining Eq. (11), (17) and (20), the Lagrangian multiplier method is introduced to get a new equation shown as follows:

$$
\begin{aligned}
& \boldsymbol{J}^{\mathrm{T}} \boldsymbol{J} \boldsymbol{k}=\boldsymbol{\lambda} \boldsymbol{M} \boldsymbol{k} \\
& \boldsymbol{k}^{\mathrm{T}} \boldsymbol{M} \boldsymbol{k}=1
\end{aligned}
$$

Moreover, since $\|\boldsymbol{J} \boldsymbol{k}\|^{2}=\boldsymbol{k}^{\mathrm{T}} \boldsymbol{J}^{\mathrm{T}} \boldsymbol{J} \boldsymbol{k}=\boldsymbol{\lambda} \boldsymbol{k}^{\mathrm{T}} \boldsymbol{M} \boldsymbol{k}=\boldsymbol{\lambda}$, the purpose of Eq. (21) is to obtain the minimum positive eigenvalue and its corresponding eigenvector.

An Ellipsoid Fitting Calibration Algorithm Based on Genetic Algorithm. Since the genetic algorithm has shown high potential in searching an optimal solution in large space ${ }^{[13]}$, an ellipsoid fitting method based on the genetic algorithm is proposed to solve the problem of the non-optimal plane equation. The genetic algorithm search the optimal intersecting plane equation, and then find the optimal positive eigenvalue and its corresponding eigenvector. The details of are shown in Fig. 1 and Table 1 respectively.

The closer the individual approaches the optimal, the greater the fitness value is. In the case of ellipsoid fitting, the objective function is smaller when the ellipsoid parameters are better. In other word, when the corresponding minimum positive eigenvalue is smaller, the data pointers can be closer to the fitting ellipsoid surface. In addition, in order to avoid the plane parameters for which genetic algorithm searches can not meet the constraint Eq. (11), the penalty function is introduced into the fitness function. Accordingly, a new fitness function is proposed as follows:

$$
S=\frac{1}{\lambda}+I
$$

where the penalty function is

$$
\begin{aligned}
& I=r \cdot p \\
& p=4 A B-C^{2}-1 \\
& r=\left\{\begin{array}{cc}
0 & p=0 \\
150 & 0<|p|<0.001 \\
500 & |p| \geq 0.001
\end{array}\right.
\end{aligned}
$$




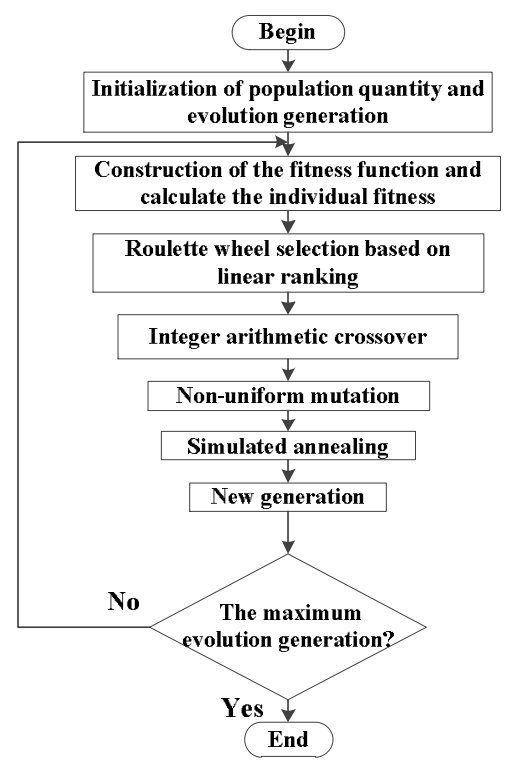

Fig. 1 Flowchart of genetic algorithm

Table 1 The parameters of genetic algorithm

\begin{tabular}{ccc}
\hline No. & Parameter & Value \\
\hline 1 & Maximum generation & 400 \\
2 & Population quantity & 600 \\
3 & Crossover probability & 0.85 \\
4 & Initial mutation & 0.2 \\
5 & probability & 50 \\
\hline
\end{tabular}

To avoid local minimal, simulated annealing algorithm is adopted in genetic algorithm, which can evolve to a better adaptation of the subgeneration ${ }^{[14][15]}$, which improves directivity of genetic algorithm. In this paper, the simulated annealing algorithm is used to screen new individuals after crossover and mutation, so that the selected individuals can evolve to the optimal individual.

Metropolis criterion is a significant part of the simulated annealing algorithm. It can be written as

$$
P_{i}=\left\{\begin{array}{cc}
1 & S(y) \geq S(x) \\
\exp \left((S(y)-S(x)) / T_{i}\right) & \text { other }
\end{array}\right.
$$

where $x=\left[\begin{array}{lll}\alpha_{1} & \beta_{1} & \gamma_{1}\end{array}\right]$ represents the parent individual. $y=\left[\begin{array}{lll}\alpha_{2} & \beta_{2} & \gamma_{2}\end{array}\right]$ is the child individual corresponding to $\boldsymbol{x}$. The corresponding fitness of them are $S(x)$ and $S(y)$ respectively. If the random number $\operatorname{rand}(0,1)$ is less than $P_{i}$, then the child individual is chosen. Otherwise, the parent is retained.

On one hand, the plane intersection method enables the constraint Eq. (11) to satisfy the condition of ellipsoid fitting. On the other hand, it makes the ellipsoid parameter only related to the plane equation. Compared with the direct search ellipsoid parameters, it can effectively reduce the search dimension, and make it easier for genetic algorithm to converge.

\section{Experiment}

In order to verify the performance of the proposed magnetic compass calibration method, the performance test of the self-developed navigation system (Fig. 2) is presented in this paper. The selfdeveloped navigation system is based on stm32f407 chip, and is equipped with ADIS16488a and P307 single-antenna GPS produced by UniStrong company. ADIS16488a is an inertial system produced by Analog devices and has a triaxial magnetic compass sensor inside. 


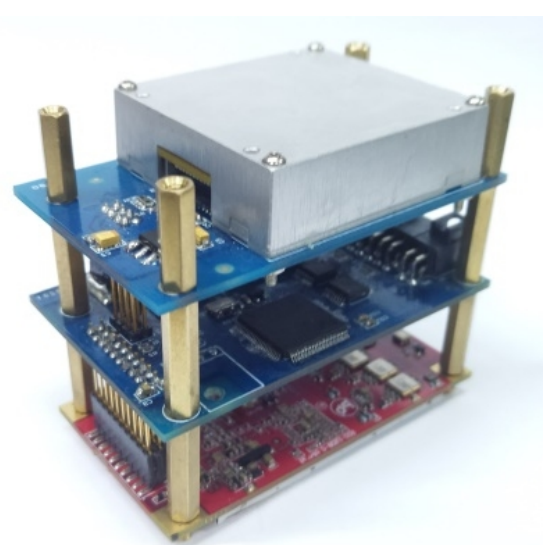

Fig. 2 Navigation system equipped with ADIS16488a

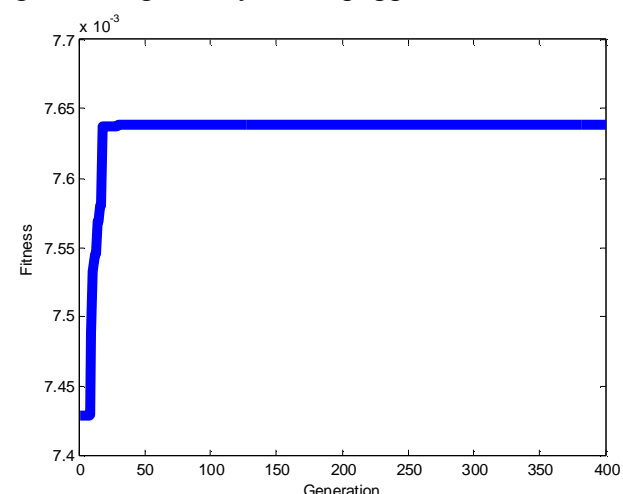

Fig. 4 Fitness function of genetic algorithm

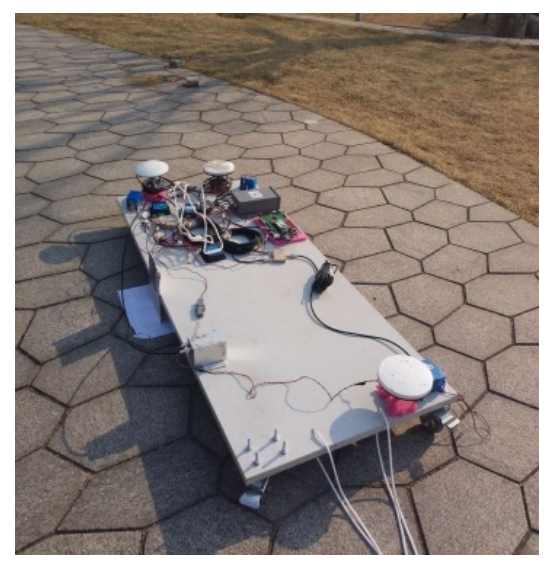

Fig. 3 Comparative experiment with dual antenna GPS

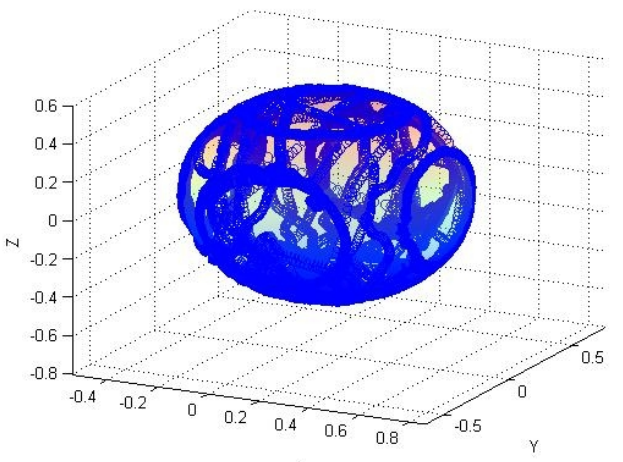

Fig. 5 Fitting ellipsoid of magnetic compass data

Table 2 Plane parameters searched by genetic algorithm

\begin{tabular}{ccc}
$\alpha$ & $\beta$ & $\gamma$ \\
\hline 0.0048 & -0.0016 & -1.0 \\
\hline
\end{tabular}

Static Experiment Based on Dual-antenna GPS. This experiment is operated on a self-developed wooden platform (Fig. 3). A pair of GPS antennas is mounted at each end of the wooden platform's long edge with a baseline of 1.6 meters. The dual-antenna GPS is UN237C, whose azimuth accuracy can reach 0.1 degree when the baseline reaches more than $1 \mathrm{~m}$. In addition, a GPS antenna is installed in the short edge of the platform for navigation system. During the experiment, the platform is rotated approximately 30 degrees each time. The magnetic azimuth output by navigation system and the GPS azimuth are recorded simultaneously.

$$
\begin{aligned}
& \boldsymbol{G}=\left[\begin{array}{ccc}
0.7483 & 0.0048 & 0.0036 \\
0 & 0.7506 & -0.0013 \\
0 & 0 & 0.7571
\end{array}\right] \\
& \boldsymbol{b}_{\mathrm{m}}=\left[\begin{array}{l}
0.1234 \\
0.0305 \\
0.0195
\end{array}\right]
\end{aligned}
$$

Before the experiment, it is necessary to calibrate the magnetic compass inside the self-developed system. With the data output by the system rotated in the 3-D space, the genetic algorithm is used to search the optimal interception plane. Benefiting from the improvement of directivity, the fitness function can converge to the optimal value rapidly (Fig. 4). The plane parameters searched are fixed values with the same data (Table 2). Then the fitting ellipsoid (Fig. 5) and calibration parameters (Eq. 25) are obtained. After the calibration parameters are introduced into the system, a comparative experiment of magnetic azimuth and dual-antenna GPS azimuth is carried out. The comparison results of the azimuth are shown in Fig. 6 and Fig. 7. From the results of Fig. 7, the maximum deviation is 0.9 
degree, and the standard deviation is 0.46 degree. It is obvious that the magnetic azimuth of the magnetic compass can achieve high precision through calibration of this method.

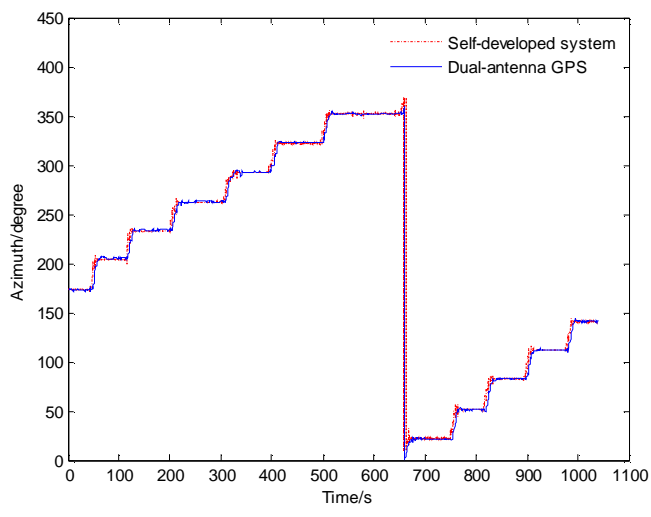

Fig. 6 Comparative results of systematic magnetic azimuth and dual antenna GPS azimuth

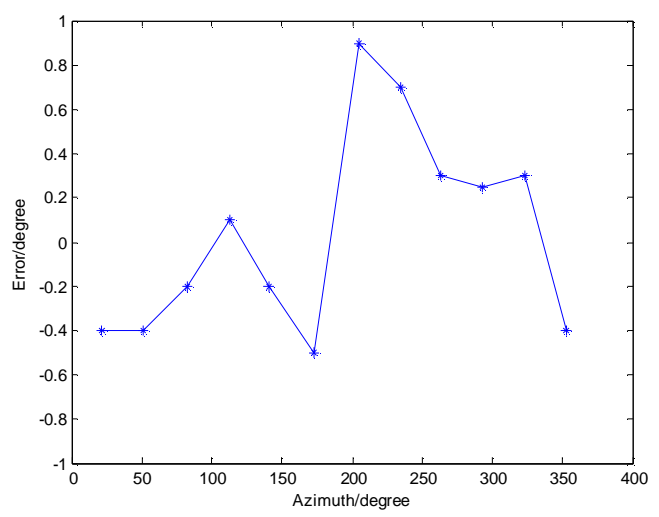

Fig. 7 Magnetic azimuth error

Dynamic Running Test. In order to further verify the system performance of the calibration, a Positioning and Orientation System (POS), whose azimuth nominal accuracy is 0.02 degree, is introduced into the dynamic running experiment as the standard azimuth reference. The self-developed navigation system and POS system are fixed in a car. The calibration parameter is written into the self-developed system and the running experiment is conducted to compare the magnetic azimuth between the self-developed system and the POS. The platform is shown in Fig. 8.

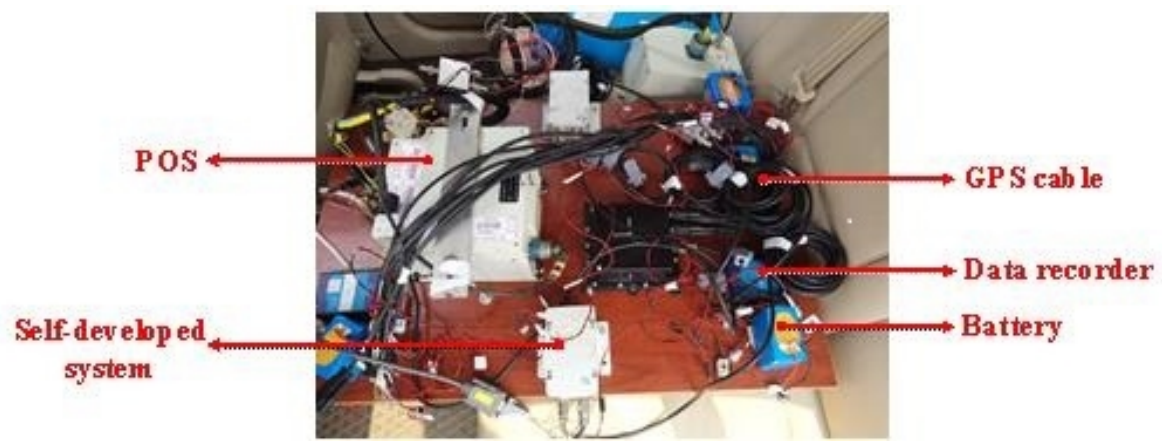

Fig. 8 Platform of running test

Through comparison of the POS system, the results of the azimuth comparison and the azimuth difference are shown Fig. 9 and Fig. 10 respectively. The azimuth difference is basically maintained within one degree, with the standard deviation of 0.68 degree. It can be concluded that the magnetic azimuth can achieve better precision in dynamic situation after calibration.

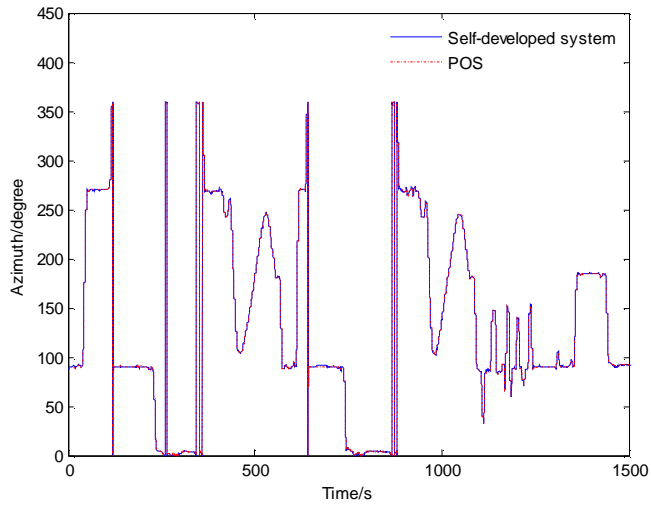

Fig. 9 Experimental data of running test

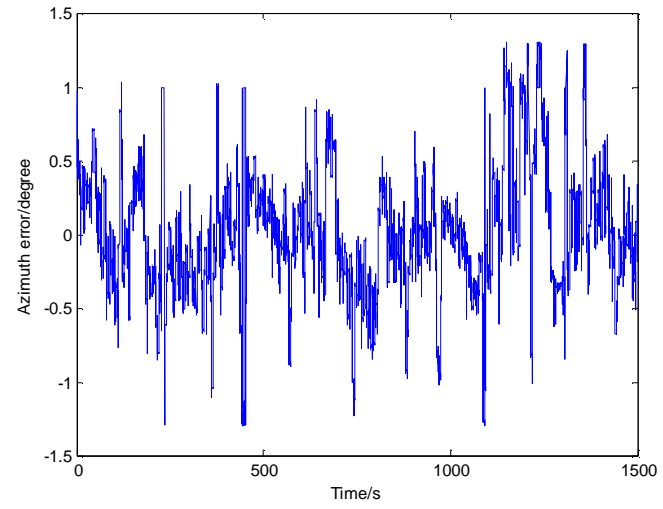

Fig. 10 The magnetic azimuth error of the running test 


\section{Conclusions}

1) This algorithm can achieve the calibration of the magnetic compass with high accuracy in both static and dynamic situations.

2) The method, which uses a plane to intercept the ellipsoid, correlates the fitting parameters of an ellipsoid equation to the parameters of the plane equation. It enables the result of the calibration directly related to the search result of genetic algorithm. The searching dimension of this method is low and easy to be implemented.

3) In the genetic algorithm, simulated annealing is introduced to deal with the problem of poor directivity. Due to the improvement of directivity, the genetic algorithm can search the optimal plane parameters, and then the optimal parameters of the ellipsoid equation are realized.

\section{Acknowledgements}

This work was financially supported by the Beijing Science and Technology Plan (D171100006217003, D161100005816001) and National Natural Science Foundation of China (61473019, 61573040, 61633002).

\section{References}

[1] Araguas G, Paz C, Gaydou D, et al: Orientation estimation fusing a downward looking camera and inertial sensors for a hovering UAV. 2013 16th International Conference on Advanced Robotics (ICAR). Vol. 131, no. 2(2013), p. 1-6.

[2] Markovic R, Krajnc A, Matko D: Calibration of a solid-state magnetic compass using angular-rate information from low-cost sensors. IET Science, Measurement \& Technology. Vol. 5, no. 2(2011), p. $54-58$.

[3] N Bowditch, in: American practical navigator, edtied by Defense Mapping Agency Hydrographic Center, Bethesda (1995), p. 201-295.

[4] Gebre-Egziabher D, Elkaim G H, Powell J D, et al: Calibration of strapdown magnetometers in the magnetic field domain. Journal of Aerospace Engineering. Vol. 19, no. 2(2006), p. 87-102.

[5] Foster C C, Elkaim G H: Extension of a two-step calibration methodology to include nonorthogonal sensor axes. IEEE Transactions on Aerospace and Electronic Systems. Vol. 44, no. 3(2008), p. 1070-1078.

[6] JaeMu Yun, Jae-Pyung Ko, JangMyung Lee: An inexpensive and accurate absolute position sensor for driving assistance. IEEE Transactions on Instrumentation and Measurement. Vol. 57, no. 4(2008), p. 864-873.

[7] Dorveaux E, Vissiere D, Martin A P, et al: Iterative calibration method for inertial and magnetic sensors. Proceedings of the 48th IEEE Conference on Decision and Control held jointly with 2009 28th Chinese Control Conference. p. 8296-8303, 2009.

[8] Pilu M, Fitzgibbon A W, Fisher R B: Ellipse-specific direct least-square fitting. IEEE Proceedings of International Conference on Image Processing. Vol. 3(1996), p. 599-602.

[9] Fang J, Sun H, Cao J et al: A Novel Calibration Method of Magnetic Compass Based on Ellipsoid Fitting. IEEE Transactions on Instrumentation \& Measurement. Vol. 60, no. 6(2011), p. 2053-2061.

[10] Grammalidis N, Strintzis M G: Head detection and tracking by 2-D and 3-D ellipsoid fitting. IEEE Proceedings of Computer Graphics International Conference. p. 221-226, 2000.

[11] Ying X, Yang L, Kong J, et al: Direct Least Square Fitting of Ellipsoids. ICPR 2012-21st International Conference on Pattern Recognition. p. 3228-3231, 2012. 
[12]Pylvänäinen T: Automatic and adaptive calibration of 3D field sensors. Applied Mathematical Modelling. Vol. 32, no. 4(2008), p. 575-587.

[13] Morris, Garrett M, Goodsell, David S, Halliday, Robert S, et al: Automated docking using a Lamarckian genetic algorithm and an empirical binding free energy function. Journal of Computational Chemistry. Vol. 19, no. 14(2015), p. 1639-1662.

[14]Li M S, Zeng P H, Zhong R W, et al: A complex-genetic algorithm for solving constrained optimization problems. 2008 International Conference on Machine Learning and Cybernetics. Vol. 2(2008), p. 869-873.

[15]Demiroz G, Yilmaz C: Using simulated annealing for computing cost-aware covering arrays. Applied Soft Computing Journal. Vol. 49(2016), p. 1129-1144. 